\title{
Editorial
}

\section{Rainbow Stars-Multi-Color Laser Emission for Science and Art}

\section{Totaro Imasaka}

Division of International Strategy, Center of Future Chemistry, Kyushu University, 744 Motooka, Nishi-ku, Fukuoka 819-0395, Japan; imasaka@cstf.kyushu-u.ac.jp; Tel.: +81-92-802-3294

Academic Editor: Takayoshi Kobayashi

Received: 19 January 2017; Accepted: 23 January 2017; Published: 4 February 2017

\section{Prologue: The Following Is a True Story}

December 28, 1987:

Shuichi Kawasaki, one of my graduate students, shouted for me to:

"Come to the laboratory, immediately!"

When I arrived, to my great surprise, I saw numerous laser spots twinkling very strongly on the screen.

"How fantastic! How could you generate such a colorful beam, today?" I asked him.

"I really don't know. I was trying to get better data for my thesis, and I maximized the output power of the laser which is introduced into pressurized hydrogen."

I thought this is a sort of nonlinear optical phenomenon, and that I might be able to enhance this colorful emission by increasing the output power of the dye laser. However, I immediately noticed that the dial indicating the voltage applied to the pump laser was already in the red zone, warning of possible, serious damage to the excimer laser.

"Shuichi! We might be able to slightly increase the output power by adjusting the emitting wavelength to the gain maximum, which might enhance this curious phenomenon."

"That is not correct, although I am not sure why. These colorful spots only appear when the wavelength is adjusted 6-7 nm away from the maximum of the gain curve." Shuichi said.

"I cannot believe it. This phenomenon can be observed only when the output power is at a maximum, indicating that it is generated by a type of nonlinear optical effect."

He moved the laser wavelength to the maximum of the gain curve, and surprisingly all of the colorful spots disappeared.

"Did you adjust the laser wavelength precisely at the center of the gain curve?"

He motioned with his eyes to look at the meter, indicating the laser power. I realized that the power definitely increased when the laser wavelength was changed.

"Unbelievable! Is this power meter working?" I asked.

Without saying anything, Shuichi interrupted the laser beam path. The needle went down to zero, indicating that the power meter was functioning correctly.

"Unbelievable! This cannot be possible! How can we explain the generation of this colorful beam? This appears through a nonlinear optical effect that requires a high level of power for more efficient generation. It appears, however, only when the laser power is decreased slightly from the maximum. This is logically inconsistent."

"Look! Totaro. I will change the laser wavelength and we can observe a continuous color change. This is so fantastic, and so beautiful."

All of the colorful spots, however, disappeared when the laser wavelength was moved far away from the optimum.

"Shuichi! Move the wavelength back in the opposite direction." "Oh! So fantastic!" 
The spots, almost equally spaced in frequency, could be clearly seen and their colors changed continuously over the entire visible region.

"Marvelous! I can see all the rainbow colors blinking like stars!"

....Shuichi and I, two researchers with no intelligence, enjoyed ourselves by looking at the dramatic color changes until late at night.....

\section{Inspiration: A Sparking of Mechanism}

When I was walking on the campus for exercise, a possible mechanism flashed through my mind: the reason why the multi-color emission is observed only when the laser wavelength is shifted 6-7 nm away from the gain maximum might be due to an amplified spontaneous emission (ASE). In the daily adjustment of the dye laser, this undesired emission, which appears at the gain maximum, needs to be reduced as much as possible. However, in the worst case, two laser emissions (i.e., an oscillating beam and ASE with a rather broad bandwidth) appear with comparable intensities. These emissions might interact with each other and produce this colorful beam, but only when the separation of the frequencies of these emissions satisfies some restriction arising from some property of the hydrogen that is used as a Raman medium. I became excited and hurried back to my office.

I asked Shuichi to measure the emission spectrum of the laser and to check the frequency separation of the oscillating beam and ASE. The value coincided exactly with the rotational Raman-shift frequency of hydrogen, verifying my above reasoning: four-wave Raman mixing-a sort of nonlinear optical phenomenon requiring a two-color beam-played an important role in the generation of numerous rotational Raman emissions. To remove the undesirable ASE, the manufacturer of the laser developed a new cavity design, but this, contrary to expectations, sometimes induced a strong ASE. In addition, unfortunately (or I should say fortunately), Shuichi's skill in aligning the laser was so poor (sorry) that he generated a two-color beam efficiently, but by accident. This complete story, including the defect in the laser, is described in a newsletter published by the manufacturer of the laser.

\section{Publication: Is Rainbow Stars the Name of a Toy?}

I was interested in publishing this finding in a scientific journal. I nicknamed this phenomenon, "Rainbow Stars", because many spots with rainbow-colors appeared when the beam was passed through a prism and projected onto a white screen. However, the reviewer of the journal to which the paper was submitted suspected the novelty of this work and suggested removing this nickname. To publish the paper, I regretted deleting this word according to the reviewer's suggestion. A few years later, Shuichi said to me, "Do you know the term "Rainbow Stars" remained in the published paper?" I immediately checked the manuscript and noticed that we forgot to delete the term "Rainbow Stars" in the figure, and the editor and reviewer did not notice. Shuichi and I smiled with pleasure (apologies to the editor and reviewer). Several years later, I found a toy named "Rainbow Stars" in the souvenir shop of a science museum in the USA, which was a set of colorful plastic plates containing a variety of phosphors to produce fluorescence/phosphorescence when irradiated with ultraviolet light. The name was registered as a trade mark, but was issued after the publication of our paper. Because of this, I have priority in using the phrase "Rainbow Stars".

\section{Art: Laser Illumination}

I was unable to find any applications for this rainbow-color laser. However, it is well known that color perception is derived from three types of cone cells in the human eye, each of which has different spectral responses, which are partially superimposed on each other. Each cone cell generates and transmits a signal, at which point the color is recognized by the balance of the signals. Alternatively, all of the frequencies of light are required for the complete reproduction of a color, as is recognized from a de I'Eclairage $x-y$ chromaticity diagram. In any case, a monochromatic multi-color laser would be desirable for the complete reproduction of a color image. It should be noted that a monochromatic light—such as a laser-easily interferes and twinkles due to the fine speckles that are induced. Such 
illusional light—which cannot be reproduced by photographic technology—is used in laser light shows. Because of this, such a multi-color laser might be widely used, e.g., in large-scale holography for outdoor use or in artistic presentations, entertainment, or amusement.

Due to the excellent color pictures in a magazine, I joined a society of lighting in Japan. I submitted a paper to an international journal sponsored by this society, in which I reported on the development of a high-power multi-color laser. The article contained a picture consisting of colorful beam patterns showing excellent performance of Rainbow Stars. When it was published, I was very disappointed to find that the picture was published as a "black-and-white" image and I withdrew my membership from this society a few years later.

I developed a design curriculum for undergraduate students. They made a variety of arts and devices for laser illumination. One such example was a "miracle clock" that consisted of three rotating rainbow-bars indicating the hour, minute, and second. Another interesting product was a picture of my face made of numerous optical fibers coupled with rainbow-color beams. I organized a laser show on the University campus, and many people came to enjoy this fantastic event.

\section{Mistake: A Phone Call-This Is a Nonfiction Story}

One day, I received a phone call.

"Hi! Totaro speaking."

"This is a phone call from the broadcast company of ....... I heard that you discovered a new phenomenon called Rainbow Stars. I would appreciate it if you could explain it to us."

I felt very lucky. I expected that our work would be introduced to many people via a television broadcast, and I became nervous and excited.

"That is not so great to call it a "discovery". I may have slightly exaggerated our research work when we wrote about it."

"Could you explain the phenomenon that you found in simple terms?"

I was afraid that he might not be able to understand terms such as "stimulated Raman scattering" or "four-wave Raman mixing", and even "tunable laser" or "spectroscopic measurement".

"Well. When we were focusing a laser beam into hydrogen, the laser accidentally emitted at two wavelengths...."

"What does "two wavelengths" mean?"

I thought that I should be more careful and not use any complicated scientific words.

"O.K. First, there were two colors, and then many colors appeared...."

However, it became difficult to explain our research while using no scientific words. I was confused myself and found it difficult to re-construct my logic for that explanation. At that moment, a good idea occurred me.

"O.K. You are working in a broadcast company, and you may understand that all the colors are reproduced by the superposition of three primary colors. Rainbow Stars can produce all of the colors directly and furthermore all of the emissions are monochromatic."

"What does "monochromatic" mean?"

I started losing my temper, but I replied while attempting to control myself.

"It means (I was meanwhile thinking) that such a laser beam can produce beautiful and twinkling light which can never be reproduced by current technologies such as television."

(Heavens!) I immediately noticed that I had made a big mistake in telling the truth, like a criminal talking with Detective Colombo.

"Thank you very much for the interesting discussion about your recent success. I hope to contact you for a more detailed discussion at a later time."

My mistake was to say that Rainbow Stars cannot be reproduced by a television, even though he wanted to give me a chance to introduce our work to many people by that medium.

Since then, no phone calls have been forthcoming from this company. 


\section{Idea: No Comments from the Reviewer}

In 1992, a new idea occurred me. Rainbow Stars can be used to generate a series of emission lines spaced by a Raman shift frequency. Such equally-spaced emission lines are produced as longitudinal modes to generate mode-locked ultrashort pulses. The major difference from a conventional mode-locked laser is its wide frequency domain and large spacing between the spectral lines. When Rainbow Stars was used, we were able to generate extremely-short optical pulses at an extremely-high repetition rate. I thought this is "of course" impossible, because many researchers would have proposed such a splendid idea if it could be accomplished. However, I was very interested in knowing why this cannot be achieved. Then I submitted a paper to a journal on this topic, although I considered to receive negative comments from the reviewer (apologies to the editor and reviewer of the journal). I expected to receive the following comments from the reviewer.

"Thank you for sending me an interesting manuscript. However, such a mode-lock laser cannot be achieved as is well-known in physics. It is my honor to explain the reason for why you are wrong. ...... I wish you great success in your research field in the future, and please accept my sincere congratulations on your efforts in this area of research."

However, I shortly received a galley proof from the journal without any editor's or reviewer's comments. I was very surprised and lost the chance to withdraw my paper. Even after its publication, I thought that such an approach would be, "of course", difficult in a real world, because we needed to generate this phenomenon using a femtosecond laser with a broad spectral bandwidth to generate a single optical pulse, although a narrow-band laser is essential for the efficient generation of stimulated Raman emissions. In fact, I could not find any paper reporting the generation of a Raman emission using a femtosecond laser.

\section{Finding: Data Thrown in a Trash Box-This Is a True Story}

One day, I said to one of my undergraduate students:

"Why don't you construct a two-color dye laser and generate rainbow-color emissions by focusing the beam into hydrogen. This would be very interesting for you."

I was waiting for the results of his efforts, but he appeared to have done nothing. Then, I asked him the reason for this disappointing effort. He replied that:

"I did my work last night and confirmed the generation of rainbow-color emissions. However, I stopped my study because the spectral bandwidth of the dye laser was broad, and you told me that the spectral bandwidth must be narrow enough to generate multi-color emissions."

I was wondering about his answer and said to him:

"This phenomenon occurs only when you use a narrow-band laser because of the narrow bandwidth of the Raman gain. Your reply seems to contradict this well-known consideration and would imply that you have not carried out the experiment."

He was slightly excited and said:

"I assure you that I did my work last night. If you don't think so, please look at the data I measured yesterday!"

"Where are the data?" I asked.

He pointed at a trash box in the laboratory, and I looked for the data. I noticed that the paper of the strip chart recorder was almost at the end of a roll and he stopped his work for this reason. However, a broad dye laser spectrum was observed, as he said. I was very surprised to see the data because he said he observed a Raman emission. If these findings were correct, it should be possible to generate Raman emissions using a femtosecond laser with a broad spectral bandwidth. However, I suspected his work was flawed in some way, and the following year I asked a new undergraduate student to construct two dye lasers in which the spectral bandwidths can be independently changed to study the effect of the spectral bandwidth on the generation of Raman emissions. Surprisingly, the Raman emissions were efficiently generated, even when two broadband dye lasers were used (I felt very sorry for the former undergraduate student). 


\section{Fantasy: Laser Display in the Exhibition}

We had no budget to continue our fundamental study, and I attempted to find applications for Rainbow Stars. On one occasion, a new technician started working in my laboratory. He had graduated from a technical high school and was 18 years old. I decided to demonstrate a multi-color laser display in an exhibition in Tokyo, which was $2 \mathrm{~h}$ from my town by airplane and $8 \mathrm{~h}$ by train. First, we encountered trouble in transporting a Raman cell that was pressurized at several atmospheres with hydrogen. We then negotiated with a local supplier to send a hydrogen-gas cylinder directly to the location of the exhibition in Tokyo. Later, we noticed that even vacuum pump oil cannot be transported because it contains a petroleum product. We spent more than $8 \mathrm{~h}$ in preparation, e.g., the adjustments of a Nd:YAG laser and other optical components for a display. We finished all the work just before the exhibition started in the morning. It was very exciting, and we enjoyed this demonstration.

I had a chance to do a laser show in Kurume City near my town (i.e., a 2-h drive by car). This was a combination of Rainbow Stars and a large-scale optical-fiber display with a size of several square meters. Several members of the laboratory started the installation of the equipment for generating rainbow-color emissions, starting at 5 p.m. for a night-time show. However, we noticed that a prism that is used for beam bending was missing, which was essential for the success of a laser show. I made a phone call to a postdoctoral fellow and asked him to bring a prism immediately from the laboratory to Kurume City by driving a car in a freeway. As a result, we were able to demonstrate Rainbow Stars at mid-night and were able to return to our laboratory by 2 a.m.

\section{Challenge: Double Rainbow!-This Is a True Story}

I had the opportunity to purchase an ultraviolet (UV) femtosecond laser which consisted of a dye laser with an excimer amplifier. I started a research program to generate rainbow-color emissions using a femtosecond laser. Based on an agreement with the manufacturer of the laser, the user was responsible for the operation and maintenance of the laser. However, the laser stopped working within a week of its installation by an engineer, who came from a foreign country. My graduate student and I attempted to make the repairs, but this proved difficult. After more than one year, we noticed that one of the optical components in the laser was poorly designed and had a problem in its structure. I pointed this out and received a newly-designed component from the manufacturer. After replacing it, we were able to immediately operate the laser. The graduate student then tried to generate Raman emissions using this UV femtosecond laser. After a few days, I asked him:

"What happened?"

"Nothing happened. I cannot even transmit the laser beam through a Raman cell."

"I think you are using borate-glass windows in the Raman cell. You should use fused-silica windows to transmit a UV beam." I said.

"I am sure that I am using fused-silica windows, and that a UV light can be transmitted through the Raman cell."

"If you are correct, please explain the reason why the UV femtosecond pulse cannot be transmitted through the cell."

"That is really puzzling to me." He said.

I was very disappointed to hear his senseless answer.

I also had an opportunity to purchase a different type of femtosecond laser-a Ti:sapphire laser that can be operated at $10 \mathrm{~Hz}$ in the near-infrared (NIR) region. An engineer of the manufacturer said that a daily start-up of the laser requires $8 \mathrm{~h}$. I asked another graduate student to generate Raman emissions using the femtosecond laser. After several months, the graduate student came to me and said.

"I was able to observe rainbow-color emissions today!"

"Marvelous! Don't touch any of the components, otherwise you will lose this phenomenon when you are attempting to optimize the equipment." I said. 
He immediately took a picture of the multi-color emissions. One of them consisted of a ring-shaped pattern, and he named this phenomenon, "rainbow ring". It was sometimes possible to observe another rainbow-color emission in the form of a streak. He named this, a "double rainbow". Surprisingly (or I would say, as naturally expected from theory), a rainbow-color emission was observed using a femtosecond laser. My graduate student and I were very excited by this finding, and were pleased with our good luck. However, the situation changed dramatically in a few weeks. The graduate student said that the ring pattern originated from an optical Kerr effect and the streak from self-focusing in the hydrogen gas. If he is correct, how can we find Rainbow Stars?

\section{Passion: Generation of Rainbow-Color Emissions-This Is a True Story}

The graduate student using the UV femtosecond laser was enthusiastically continuing his work. Finally, he found the reason for why the UV pulse did not transmit through the Raman cell. It was due to the two-photon absorption of the high-intensity UV femtosecond pulse by the fused silica window. He then expanded the beam at the window and passed through the femtosecond beam. He immediately observed rainbow-color emissions, and I was very excited about this result. However, vibrational and rotational Raman emissions were generated simultaneously, and it was difficult to exclusively generate equally-spaced rotational lines.

Another graduate student using the Ti:sapphire laser was also concentrating his efforts on generating multi-color emissions. He shouted for me:

"Today, I was able to generate rotational Raman emissions!"

"Are you sure this time?" I said.

"Absolutely, I am sure. I observed numerous twinkling spots in line when the beam was passed through a prism and projected onto a screen. They were equally spaced in frequency, and were extended from the far-UV to the NIR! I was wondering whether I should call you immediately to look for yourself or not."

"When did you find them?"

"Two o'clock", he said.

"AM or PM?"

"AM", he answered.

(I thought I was happy not to have received his phone call at such an early time.)

"How were you able to obtain this result?" I asked to him.

"Today, I chirped the laser pulse for stretching."

Later, we noticed that the laser was operating under improper conditions: the laser had a slightly narrow spectral bandwidth (longer pulse width), although the engineer from the manufacturer had suggested that we should not operate the system under such unfavorable conditions because this might cause serious damage to the laser. In fact, we frequently destroyed expensive Ti:sapphire rods in the experiment. However, such a condition was favorable for suppressing undesirable effects such as self-phase modulation and self-focusing. We also noticed that the NIR laser was useful for the exclusive generation of rotational Raman emissions.

\section{Dream: Molecular-Optic Modulator}

One day, I was thinking about a laser cavity. I noticed that a laser consisting of equally-spaced frequencies can transmit through the cavity, and that the intensity of the light can be strongly enhanced. If this is correct, all rotational Raman emissions can be resonated in the cavity under certain conditions. This consideration suggests that Rainbow Stars-a type of nonlinear optical effect-can be generated even using a continuous-wave $(\mathrm{CW})$ low-intensity laser. This idea was published in a scientific journal and was also patented. In order to increase the intensity of the light in the cavity, it is necessary to increase the finesse of the cavity using a pair of mirrors with a high reflectivity (e.g., $99.98 \%$ ), and to use a monochromatic laser beam with a narrow linewidth (e.g., $100 \mathrm{kHz}$ ). We solved numerous problems, and were able to match the laser beam and the cavity after a 5-year effort! Eventually, 
we were able to generate a Stokes beam using a CW Ti:sapphire laser. An optical beat formed by the fundamental beam, and the Raman beam was measured using a laboratory-made autocorrelator. Based on current technology, it is possible to modulate a laser beam in the $\mathrm{MHz}$ region using an acousto-optic modulator (AOM) and in the $\mathrm{GHz}$ region using an electro-optic modulator (EOM). We were able to modulate the laser beam in the $\mathrm{THz}$ region based on molecular motion. We referred to this device as a "molecular-optic modulator (MOM)".

In order to generate a train of pulses, it is necessary to generate more than three emission lines. By carefully adjusting the laser wavelength and the cavity length, it was possible to generate the second Stokes beam, in addition to the fundamental and the first Stokes beam. A train of optical pulses, generated at $17.6 \mathrm{THz}$, was measured using the autocorrelator.

\section{Obsession: Dispersion-Compensation in the Cavity}

The spacing among the three emission lines which consist of the fundamental and two Stokes beams cannot be exactly equal, because the refractive index changes with changing wavelengths. For this reason, the waveform of the pulse changes at different times. In order to solve this problem, it is necessary to use a coherent process such as four-wave Raman mixing (FWRM). However, generating an anti-Stokes beam by FWRM is a difficult task due to unavoidable positive dispersion of hydrogen in the cavity. To address this issue, we developed a dispersion-compensated optical cavity for anti-Stokes generation. A pair of mirrors with negative dispersions were used, and the total dispersion in the cavity was adjusted to zero by optimizing the hydrogen pressure and by adding a noble gas with different optical properties. This was not an easy task, because the cavity length, the laser wavelength, and the pressures of the hydrogen and xenon gases must be very carefully changed in a stepwise manner and be precisely optimized. A graduate student did his best, and was eventually successful. After 4 years, he found the conditions needed to generate an anti-Stokes beam; the hydrogen gas pressure should be optimized at $530 \pm 5 \mathrm{kPa}$ at a xenon pressure of $140 \mathrm{kPa}$ !

In order to generate a train of the fastest optical pulses, it is necessary to generate a three-color beam with the largest frequency separation. When we use a three-primary-color laser, it would be possible to generate the highest repetition rate using an optical (visible) beam. Such a laser was generated using the second harmonic emission ( $532 \mathrm{~nm}$, green) as a fundamental beam to generate the Stokes beam $(683 \mathrm{~nm}$, red) and the anti-Stokes beam $(436 \mathrm{~nm}$, blue), which supports the generation of a $125-\mathrm{THz}$ pulse train. If necessary, a three-primary-color laser with better visibility could be generated using deuterium $(459 \mathrm{~nm}, 532 \mathrm{~nm}, 632 \mathrm{~nm})$.

\section{Epilogue: What Are Rainbow Stars?}

A wide frequency domain is essential for the generation of short optical pulses, as is known from the uncertainty principle. The generated multi-color laser emissions extend from the far-UV to the NIR, and might represent an approach for a breakthrough in the generation of ultrashort pulses beyond a 1-fs barrier. In fact, a sub-cycle optical pulse can be generated by this technique, which would be impossible to achieve using current technology such as high-harmonic generation producing attosecond pulses in the extreme-UV region. Therefore, an ultrashort laser pulse generated by Rainbow Stars could have a significant impact and result in scientific breakthroughs. On the other hand, it is possible to generate ultrashort and highly-repetitive optical pulses by focusing a CW laser beam into a cavity containing a Raman medium. By phase-locking in the process of FWRM, the repetition rate and the temporal pulse profile can be stabilized by reducing the phase mismatch, thus enhancing the Raman emission. In fact, a standard of the highly-repetitive optical pulses would be obtained by this technology. A three-primary color laser would be interesting not only for use in a laser display, but also for generating the fastest-repetition optical pulses. Thus, this technology has the potential for use in ultra-high-speed data communication. 
Rainbow Stars were introduced more than 40 times in newspapers, magazines, on television, and on the front covers of textbooks. There are many funny but real stories about this, but I cannot disclose all of them in written form. I hope that I will have the opportunity to discuss this with many people in the future. 